\title{
MODIFIED LAPLACIAN FILTER AND INTENSITY CORRECTION TECHNIQUE FOR IMAGE RESOLUTION ENHANCEMENT
}

\author{
Day-Fann Shen, Chui-Wen Chiu, Pon-Jay Huang \\ Department of Electrical Engineering, \\ National Yunlin University of Science and Technology, Douleo 640, TAIWAN \\ E-mail: shendf@yuntech.edu.tw
}

\begin{abstract}
By analyzing the deterministic relationship between the lowerresolution and the corresponding higher resolution images, we propose two core techniques namely MLF (Modified Laplacian Filter) and IC (Intensity Correction) for image resolution enhancement, by which the image size can be increased revealing better details of the image contents. The simple $3 \times 3$ MLF is designed for properly restoring the frequency components attenuated in the averaging and down-sampling degradation process. The IC process iteratively refines the image quality for any resolution enhanced (enlarged) image. Experiments show that the proposed techniques can effectively improve the image qualities than bilinear or bicubic interpolation alone. It outperforms other recently developed algorithms both in perceptual quality (especially in the texture areas) and in objective quality in terms of PSNR. Both MLF and IC are simple in computations, which is quite desirable in many time sensitive applications.
\end{abstract}

Keywords: image resolution enhancement, image enlargement, super-resolution (SR), Modified Laplacian Filter (MLF), Intensity Correction (IC).

\section{INTRODUCTION}

Images of higher resolutions are highly desirable in many applications, such as medical imaging, law-enforcement, satellite imaging. However, there are fundamental limitations on acquiring high-resolution images by simply increasing the image sensors[1]. Alternately, the image resolution can be increased to exceed the hardware limitation through image-processing algorithms, which are normally referred to as (still) image super-resolution (SR) or simply image enlargement.

In most SR algorithms [2-9], the interpolation technique (bilinear, bi-cube etc.) is the fundamental technique to obtain the extra pixels, however, the interpolation alone normally generates blurry images especially in the edges and texture areas. Many researchers have developed edge-preserving techniques in order to remove the annoying blurry and blocky edges. Li and Orchard [2] proposed a new edge-directed interpolation algorithm to interpolate pixel values based on the local covariance coefficients. To reduce the complexity in [9], Huang et al. [3] classified pixels in the image into homogenous areas and edges; the edge pixels are then interpolated based on the edge direction. Shi et al. [4] used the Canny Edge detector to locate and interpolate the edges; furthermore, they modified the neighboring pixels around the edges to make the edges look sharper. The above-mentioned techniques, which focus on improving the annoying blurry and blocky edges, are referred to as the edge-enhancement approach. The training-based or learning-based approach [15-20] is another approach, by enhancing both edged and textures. Given a training set, the algorithm learns the fine details that correspond to various image regions seen at a low-resolution image, and then uses the learned relationships to predict fine details in the higher-resolution images. Freeman et al. [5,6] claimed a fast and simple one-pass training-based algorithm for creating plausible high frequency details in the zoomed images. Hertzmann et al. [7] also used a training-based method to perform super-resolution, in the context of analogies between images. Baker and Kanade [8] focused on enlarging images of a known model class, such as faces.

In many serious applications, such as medical, satellite, aerial, law-enforcement, space probing, etc., the detailed contents of a scene are even more interesting than the artificial sharp looking. In this paper. Our analysis shows that the high frequencies components are attenuated by the averaging process in the digital image acquisition process. Thus, our goal is to properly recover these suppressed high frequency components (details and edges), which in turn may

improve the blurry phenomenon due to up-sampling and bilinear (or bi-cubic) interpolation in the enlarging process.

Based on the above-mentioned analysis, we derive a modified $3 \times 3$ spatial Laplacian filter, which we refer to as the Modified Laplacian Filter (MLF) to properly restore the mid to high frequency components in the input low resolution (LR) image; it is noted that the direct inverse filtering to the degradation process may improperly amplify the noises and the aliased frequency components. The MLF filtered image is then bilinear (or bi-cubic) interpolated as initial higher resolution (HR) image. We also exploit the ideal relationship between the input LR image and the corresponding HR image, an Intensity Correction (IC) process is derived for correcting the initial HR image toward the ideal relationship. The IC process can be applied iteratively for increasing the objective image quality at the cost of more computations.

Experiments show that the proposed MLF+Bilinear+IC approach can effectively improve the image quality both objectively (PSNR) and subjectively for all test images. Furthermore, the proposed algorithm is relatively simple in computation, thus, is quite desirable to many real-world applications.

The paper is organized as follows. Section 2 briefly defines the SR problem. Section 3 analyzes the SR problem and derives the proposed algorithm. Experiments and performance comparisons are presented in Section 4. Further improvements by 
bi-cubic and iterative IC process are presented in section 5. Finally, Section 6 concludes the paper.

\section{THE SUPER-RESOLUTION PROBLEM}

The original high-resolution (HR) image is normally not available for the performance evaluation In many practical applications. Thus, the resulting super-resolution (SR) image can only be evaluated subjectively. However, it would be more convenient to have the original HR image available for the comparison and evaluation of various SR algorithms. In this paper, we formulate and simplify the SR problem as follows:

Given a $\mathrm{N}_{2} \times \mathrm{M}_{2}$ high-resolution image (original HR), obtain the corresponding $N_{1} \times M_{1}\left(N_{1}=N_{2} / 2, M_{1}=M_{2} / 2\right)$ lowresolution image (LR) by applying the degradation process. The $S R$ algorithm is to derive the best approximation both subjectively and objectively of the original HR from the $L R$.

The degradation process in the image acquisition device is shown in Figure 1.

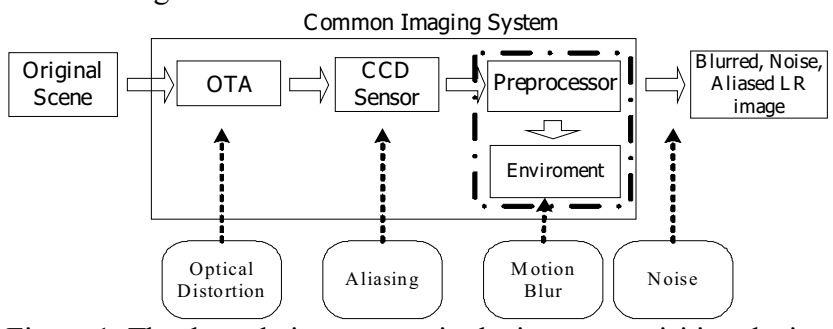

Figure 1. The degradation process in the image acquisition device

\section{THE SUPER-RESOLUTION ALGORITHM}

\subsection{The Analysis}

We derive the ideal relationship between high and low density CCD sensor module in Figure 1 as follows:

$$
f_{L R}(m, n)=\frac{f_{H R}(m, n)+f_{H R}(m, n+1)+f_{H R}(m+1, n)+f_{H R}(m+1, n+1)}{4}(\mathrm{Eq} .1)
$$

Where $f_{L R}(m, n)$ and $f_{H R}(m, n)$ are the intensity of a lowresolution pixel and its four corresponding high resolution pixels $(\mathrm{k}=1)$. For simplicity without losing generality in the analysis, we consider the two cascaded processes that converting 1-D discrete HR signal $x[n]$ of length $2 \mathrm{~N}$ to its LR version $z[n]$ of length $\mathrm{N}$,

$P_{1}$ : The averaging process

$y[n]=x[n] * h[n]$ and $h[n]=\frac{d[n]+d[n-1]}{2}$

$d[n]$ is the impulse function, i.e.

$d[n]=\left\{\begin{array}{l}1, \quad \text { for } n=0, \\ 0, \quad \text { otherwise; }\end{array}\right.$ and

$P_{2}$ : The sub-sampling process

$$
z[\mathrm{n}]=y[2 n+1], \text { for } n=0,1,2, \cdots, N-1 .
$$

In most practical conditions, the sub-sampling process $P_{2}$ is noninvertible, for recovering the high-resolution signal, the spatial bilinear (or bi-cubic) interpolation is commonly used to estimate the additional samples. For the inverse of $\mathrm{P}_{1}$ process, let $g[n]$ be the impulse response of the inverse $\mathrm{P}_{1}$ process, where $g[n]$ can be obtained by solving the simple equation $h[n] * g[n]=\delta[n]$, which is an inverse filtering problem. We apply the frequency domain analysis to find the solution, that is, $G(\omega)=1 / H(\omega)$, where $G(\omega)$ and $H(\omega)$ are the Fourier transforms of $g[n]$ and $h[n]$ respectively. We have:

$$
H(\omega)=1+e^{j \omega}=e^{-j \frac{\omega}{2}} \cos \left(\frac{\omega}{2}\right)
$$

Thus,

$$
G(\omega)=\frac{1}{H(\omega)}=e^{j \frac{\omega}{2}} \sec \left(\frac{\omega}{2}\right)
$$

The Eq.2 gives us an important insight that frequency components in the original HR image still exist in its degraded LR version. However, they are attenuated accordingly by $H(\omega)$ and contaminated by the aliasing.

Two relatively naïve attempts to recover the attenuated components by (1) Direct inversing with the 2-D version of Eq.3 and (2). The 2-D Laplacian filtering in Eq.4 is shown in Figure 2, both generate unacceptable quality.

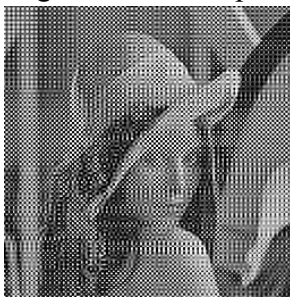

(a) Direct Inversing

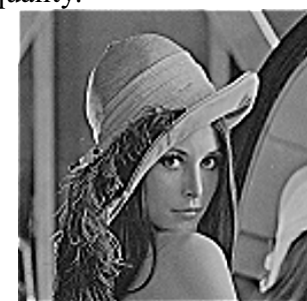

(b) Laplacian Filtering
Figure 2 the experimental results of Lena 256x256 (a) Direct Inversing (Eq.3) PSNR only 6.5B (b) Laplacian filter (Eq. 4) + Bilinear, $\mathrm{PNSR}=19.8 \mathrm{~dB}$

$$
g_{2}[m, n]=\left[\begin{array}{lll}
0 & 0 & 0 \\
0 & 1 & 0 \\
0 & 0 & 0
\end{array}\right]+\left[\begin{array}{ccc}
0 & -1 & 0 \\
-1 & 4 & -1 \\
0 & -1 & 0
\end{array}\right]=\left[\begin{array}{ccc}
0 & -1 & 0 \\
-1 & 5 & -1 \\
0 & -1 & 0
\end{array}\right]
$$

\subsection{The Modified Laplacian Filtering (MLF)}

Observation shows that the naïve Laplacian filtering by Eq. 4 yields only $19.8 \mathrm{~dB}$, however, it does enhance (too strong) the edges and textures. For the possibility of further improvements, we proceed to analyze the impulse response of the 1-D Laplacian filter: $g_{L}[n]=d[n]+(-d[n-1]+2 d[n]-d[n+1]) \quad$. Its frequency response $G_{L}(\omega)=1+2(1-\cos \omega)$, is shown in Figure 3 (with $\mathrm{A}=1)$. Note that the $G_{L}(\omega)$ deviates significantly from $\sec (\omega)$ (the inverse filter). Our strategy is to modify $G_{L}(\omega)$ so that it approximates the $\sec (\omega)$ (the inverse filter) for the mid and the lower frequency range, while provides moderate gains for the higher frequencies to avoid amplifying too much on the aliased components and noises. By introducing a parameter " $\mathrm{A}$ " as the gain attenuator, we have:

$$
G_{L A}(\omega)=1+\frac{2(1-\cos \omega)}{A} .
$$

The frequency response of $G_{L A}(\omega)$ matches the $\sec (\omega)$ curve quite well for $\omega<1.5$ and moderates gains for higher frequencies, if we choose $A=6$, as shown in Figure 3. Then,

$$
g_{L A}[n]=d[n]+\left(\frac{-d[n-1]+2 d[n]-d[n+1]}{A}\right)
$$

Experiments show that the $\mathrm{G}_{L A}(\omega)$, which has much lower gains than the $\sec (\omega / 2)$ for $\omega>1.5$, still produces degradations due to the fact that the aliased components are amplified. In order to reduce such degradations, we apply $G_{L A}(\omega)$ to the LR image right before the up-sampling and the bilinear interpolation, such 
that these over-amplified components are suppressed by the bilinear operation.

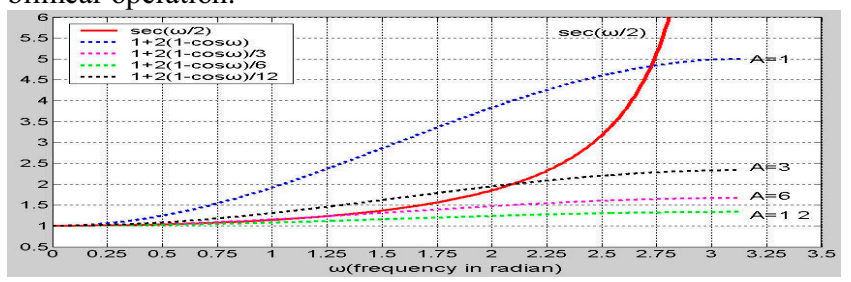

Figure 3. $\sec (\omega)$ approximated by $\mathrm{G}_{L A}(\omega)$ with $\mathrm{A}=1,3,6$ and $12, \mathrm{~A}=6$ best matches $\sec (\omega)$ for $\omega<1.5$.

We modify the 2D Laplacian filter by introducing a filter gain attenuator "A". The proposed MLF is as follows, $g_{L A}(m, n)=\left[\begin{array}{lll}0 & 0 & 0 \\ 0 & 1 & 0 \\ 0 & 0 & 0\end{array}\right]+\frac{1}{A}\left[\begin{array}{ccc}0 & -1 & 0 \\ -1 & 4 & -1 \\ 0 & -1 & 0\end{array}\right] \quad$ (Eq.7)

\subsection{Intensity Correction (IC)}

Eq. 1 models the ideal relationship between a LR pixel and the corresponding 4 pixels in the original HR image. By exploiting Eq.1, the initial SR image after MLF and bilinear interpolation can be compensated toward the original $\mathrm{HR}$ by a process called intensity correction (IC). We define the following terms before describing the IC process.

$f_{H R}(m, n)$ : The original HR image.

$f_{L R}(m, n)$ : The LR image obtained by Eq. 1 from $\operatorname{HR} f_{H R}(m, n)$.

$g_{S R}(m, n)$ :The initial SR image by (MLF+Bilinear) from $f_{L R}(m, n)$ before the IC process.

$g_{L R}(m, n)$ : The LR image derived from $g_{S R}(x, y)$ through Eq. 1 by replacing HR with $g_{S R}(m, n)$.

$e_{L R}(m, n)$ : The difference image $g_{L R}(m, n)-f_{L R}(m, n)$.

$e_{S R}(m, n)$ : Obtained by applying up-sampling and the bilinear (or bicubic) interpolation to $\mathrm{e}_{\mathrm{LR}}(m, n)$.

$g_{I C}(x, y)$ : The SR image after applying the IC correction on $g_{S R}(x, y)$.

The intensity correction (IC) process is described as follows. Given $f_{L R}(x, y)$ and $g_{S R}(x, y)$ :

Step 1: Obtain $g_{L R}(x, y)$ by Eq. 1 from $g_{S R}(x, y)$.

Step 2: Obtain $e_{L R}(x, y)=g_{L R}(x, y)-f_{L R}(x, y)$.

Step 3: Obtain $e_{S R}(x, y)$ by applying up-sampling and the bilinear (or bi-cubic) interpolation.

Step 4: The SR image after IC $g_{I C}(x, y)=g_{S R}(x, y)-e_{S R}(x, y)$

\subsection{The Proposed Super-Resolution Algorithm}

For gray-level LR image $f_{L R}(x, y)$, the proposed SR algorithm:

Step 1 (MLF+BI): Obtain $g_{S R}(x, y)$ by applying MLF to $f_{L R}(x, y)$ followed by bilinear interpolation (BI). Step 2 (IC): Obtain SR image $g_{I C}(x, y)$ by the Intensity Correction (IC) process. Step 1 and 2 can be summarized as (MLF+BI+IC).

For color images, the proposed SR algorithm is described below: Step 1: Converts the input LR color image from the RGB domain to the $\mathrm{YCbCr}$ domain. Step2: For the $\mathrm{Y}$ component, apply $(\mathrm{MLF}+\mathrm{BI}+\mathrm{IC})$ to obtain the super resolution $\mathrm{Y}_{S R}$. Step3: For the $\mathrm{Cr}$ and the $\mathrm{Cb}$ components, apply only bilinear interpolation (BI only, no MLF and IC) to obtain the super-resolution $\mathrm{Cb}_{S R}$ and $\mathrm{Cr}{ }_{S R}$. Step4: Complete the process by converting $\mathrm{Y}_{S R} \mathrm{Cb}_{S R} \mathrm{Cr}_{S R}$ back into the RGB domain.

\section{EXPERIMENTS AND EVALUATIONS}

\subsection{SR Performance Evaluation Criteria:}

Two criteria are adopted to evaluate the performance of various SR algorithms, that is, the subjective (perceptual) and objective quality of the reconstructed SR image. For color images, $\mathrm{PSNR}_{\text {Color }}=\frac{\left(4 \times P S N R_{Y}+P S N R_{U}+P S N R_{V}\right)}{6}$.

\subsection{Determination of Attenuator A}

The value of attenuator A is determined based on PSNR with 4 test images (Satellite 1026x1024, Moon 463x538, Lena 512x512 and Baboon Color, 256x256). The best A values are slightly varying from images, we recommend a fixed $\boldsymbol{A}$ value of 16 (power of 2) and is used in the rest of the paper. We also observe that it is MLF that improves the visual quality and it is IC that improves the PSNR. The computation time (MATLAB) required by the proposed $(\mathrm{MLF}+\mathrm{BI}+\mathrm{IC})$ is twice of $\mathrm{BI}$ alone, but only about $1 / 7$ and $1 / 10$ of CIE and FEI respectively.

\subsection{SR Image Quality Evaluations}

In this Section, we compare and evaluate both the objective and the subjective image quality of the 4 test images generated by the proposed SR algorithms. The objective image quality is measured by PSNR, while the subjective quality is judged by best human efforts on examining the contents of zoomed partial images.

Table 1 show that the proposed algorithm achieves significant improvements in PSNR than BI or bi-cubic interpolation only. Table 1 also shows that the proposed algorithm can be used together with more sophisticated Bi-cubic (BC) interpolation for better performance than with bilinear interpolation, at the cost of more computations. The proposed MLF $+\mathrm{IC}$ can be used together with other interpolation techniques.

Figure 4(a) is the input LR image; Figure 4(b) is the partially zoomed image in original HR image as the golden reference for perceptual examination. Figure 4(c) is the result by MATLAB bilinear interpolation alone and Figure 4(d) is the results by the proposed SR algorithm, which provides the best approximation of the golden reference in Figure 4(b) and results in sharper looking in the texture area. Figure 4(e)(f) shows error images between the SR images and the original Moon image. The restored SR image by the proposed SR algorithm yields the smallest error image than BI alone. Although the improvement in PSNR for test image baboon is trivial, the perceptual improvement is significant!

Table 1 Comparison of PSNR between bilinear and bi-cubic interpolation

\begin{tabular}{|c|l|l|}
\hline $\begin{array}{c}\text { SR Algorithms } \\
\text { Test Images }\end{array}$ & BI/ MLF+BI+IC & BC/MLF+BC+IC \\
\hline Moon & $37.0 / 41.2 \mathrm{~dB}$ & $38.3 / 42.3 \mathrm{~dB}$ \\
\hline Lena & $32.9 / 34.8 \mathrm{~dB}$ & $33.8 / 35.2 \mathrm{~dB}$ \\
\hline Satellite & $25.47 / 26.9 \mathrm{~dB}$ & $26.3 / 27.21 \mathrm{~dB}$ \\
\hline Baboon (color) 256 & $32.37 / 32.68$ & $33.29 / 33.43$ \\
\hline \\
(3) \\
\end{tabular}


(a) Moon 269x232, 8bits, gray (input LR)

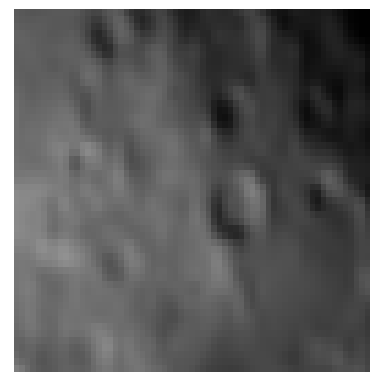

(c) Bilinear

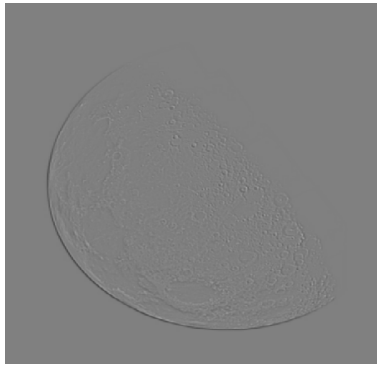

(e) Error by Bilinear

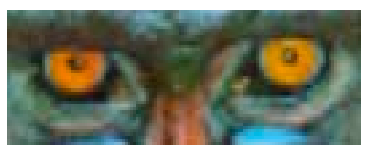

(c) Partial by BI

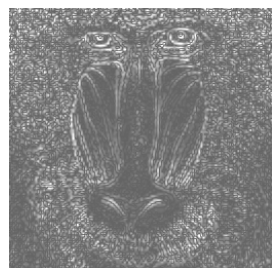

(e) Error by BI (b) The zoomed partial image in the original Moon $(538 \times 464)$

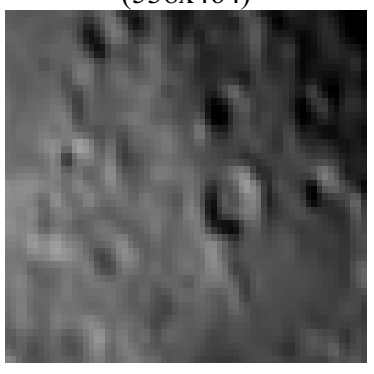

(d) MLF+BI+IC (Proposed)

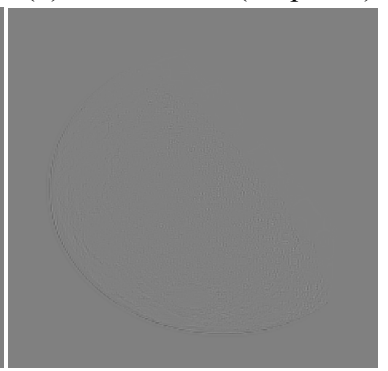

(f) Error by MLF+BI+IC (Proposed)

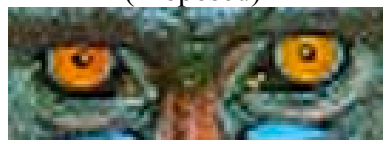

(d) Partial by Proposed

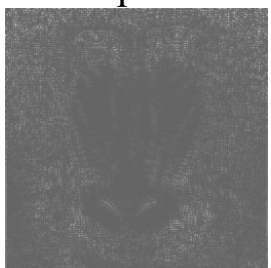

(f) Error by Proposed

Figure 4. The subjective comparison of Moon/Baboon partial zoomed and error images

Further improvements in PSNR can be achieved by applying the IC process iteratively for 2 3 time and PSNR saturated thereafter as shown in Figure 5.

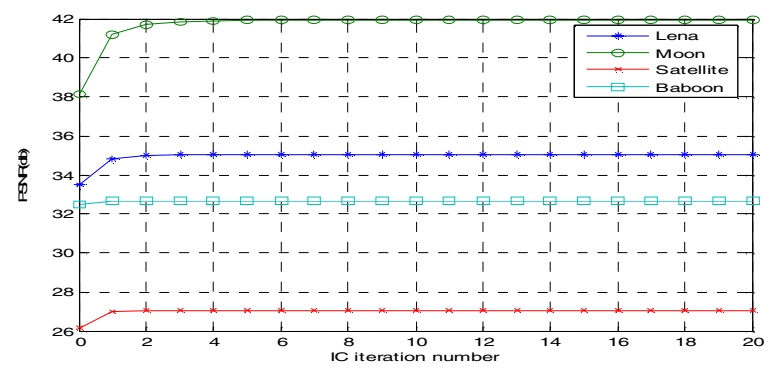

Figure 5 Effects of applying IC iteratively.

\section{CONCLUSIONS}

By analyzing the relationship between an LR image and its corresponding HR image, we derive MLF and IC which forms the core techniques in the propose MLF+BI+IC algorithm for superresolution enhancement. Compared to bilinear interpolation alone, the proposed algorithm restore the attenuated high frequency components properly, thus yields better image quality both PSNR and in perception. Furthermore, the proposed algorithm is quite simple in computation, which is highly desired in many real-world applications.

\section{ACKNOWLEDGEMENT}

This paper is supported by Taiwan Science Council under research project NSC94-2213-E-224-012.

\section{REFERENCES}

[1] Sung Cheol Park, Min Kyu Park, and Moon Gi Kang, "Super-resolution image reconstruction: a technical overview," IEEE Signal Processing Magazine., vol. 20, pp. 21-36, Mar. 2003.

[2] Xin Li, M. T. Orchard, "New Edge-Directed Interpolation," Image Processing, IEEE, vol. 10, pp. 1521-1527, Oct. 2001.

[3] C.H. Huang, M.J. Chen, C.T. Hsu, "Fast Edge-Oriented Image Interpolation Algorithm," 2003 Workshop on Consumer Electronics, Nov. 2003, Taiwan.

[4] H. Shi, R. Ward, "Canny edge based image expansion," IEEE International Symposium on Circuits and Systems, vol. 1, pp. I-785-I-788, May 2002

[5] Freeman, W.T., Jones, T.R., Pasztor, E.C., "Example-based super-resolution," Computer Graphics and Applications, IEEE, Volume: 22 , Issue: 2 , March-April 2002.

[6] W.T. Freeman, E.C. Pasztor, and O.T. Carmichael, "Learning Low-Level Vision,” Int'l J. Computer Vision, vol. 40, no. 1, Oct. 2000, pp. 25-47.

[7] A. Hertzmann et al., "Image Analogies," Computer Graphics (Proc. Siggraph 2001), ACM Press, New York, 2001, pp. 327-340.

[8] S. Baker and T. Kanade, "Limits on Super-Resolution and How to Break Them," Proc. IEEE Conf. Computer Vision and Pattern Recognition (CVPR), vol. II, IEEE CS Press, Los Alamitos, Calif., 2000, pp. 372-379. 